\title{
Consideraciones sobre el rol del pastoralismo móvil en el proceso de urbanización en el sur del Levante durante el Bronce Antiguo
}

\author{
(4) Ezequiel Cismondi \\ Universidad de Buenos Aires, Argentina
}

Fecha de recepción: 25 de julio de 2021. Fecha de aceptación: 18 de agosto de 2021.

\begin{abstract}
Resumen
El proceso de urbanización en el sur del Levante implicó cambios significativos en las poblaciones sedentarias de la región durante el Bronce Antiguo. Asimismo, se observan modificaciones en el registro arqueológico de las zonas áridas habitadas por pastores móviles, aparentemente vinculado a la aparición de mercados urbanos. Se cree que el desarrollo de las economías pastoriles estuvo relacionado con las nuevas condiciones económicas, lo que habría estimulado el desarrollo de intercambios de bienes. El objetivo central de este estudio es analizar la influencia que tuvieron los asentamientos urbanos sudlevantinos en las poblaciones móviles de las regiones áridas, y viceversa, especialmente en relación con la participación de estas últimas en las redes de intercambio, necesarias para que se lleve a cabo la "revolución urbana". Para ello, revisamos el registro arqueológico de algunos sitios asociados a grupos pastoriles para luego proponer algunas consideraciones respecto a las influencias mutuas entre las poblaciones móviles y las urbes. Consideramos que el rol de los pastores fue relevante, ya que los intercambios que se gestaron posibilitaron la acumulación de los bienes necesarios para el proceso de urbanización.
\end{abstract}

Palabras clave: pastoralismo móvil, sur del Levante, intercambios, urbanización

Considerations on the Role of Mobile Pastoralism in the Urbanization Process in the Southern Levant during the Bronze Age

\author{
Abstract \\ The urbanization process in the Southern Levant implied a significant change \\ for the sedentary population during the Bronze Age. Additionally, modifications
}


have been observed in the archaeological record in the arid zones inhabited by mobile populations, apparently linked to the appearance of urban markets. It is believed that the development of the pastoralist economy was related to new economic conditions, which could have stimulated in its stead the development of goods exchanges. The main goal of this article is to analyze the influence that the Southern Levant urban settlement had on the populations of the arid zones, and vice versa, especially the relationship to the participation of the latter in the exchange networks, necessary for the "urban revolution" to take place. To do this, a review of the archaeological record of some sites associated with pastoral groups is presented, and then, some considerations about the mutual influences between mobile population and the urban settlements are proposed. The exchange that took place made possible the accumulation of the necessary goods for the urbanization process to be possible.

Keywords: mobile pastoralism, southern Levant, exchanges, urbanization

\section{Introducción}

El proceso de urbanización, iniciado a partir de la denominada "revolución urbana", ${ }^{1}$ se desarrolló durante los comienzos del Bronce Antiguo ${ }^{2}$ IB en el sur del Levante. Este fenómeno conllevó cambios en los patrones de asentamiento de las poblaciones que habitaban la región, incluyendo la aparición de ciertas edificaciones particulares, las cuales podrían indicar la existencia de funciones sociopolíticas diferenciadas, e incluso jerarquías sociales. Además, incrementó cuantitativamente la cantidad de bienes provenientes de regiones lejanas, lo que da cuenta de la intensificación de la circulación de bienes en la región (cf. Milevski, 2010).

El elemento más distintivo de este proceso es evidentemente la aparición de asentamientos diferentes a los preexistentes. Podemos definir estos sitios como "un asentamiento rodeado por una muralla en cuyo interior se erigen edificios públicos y estructuras dedicadas a la vivienda y/o a las actividades productivas, y que además presenta un tamaño mayor que otros asentamientos -sin estas características- de la misma época y región" (Jaruf, 2019: 8). Entre los principales estudios comparativos ${ }^{3}$ respecto a los primeros urbanismos, destacan una serie de semejanzas sociales, que pueden englobarse en una mayor división social del trabajo, división entre el campo y la ciudad, estratificación social, la aparición de grupos especialistas full-time dedicados a una determinada actividad, institucionalización de las relaciones políticas

1 La revolución urbana es un concepto acuñado por Gordon Childe (1950) para definir el desarrollo de las primeras ciudades a partir de la aparición de ciertos rasgos distintivos en los asentamientos sedentarios. Entre ellos podemos mencionar una mayor extensión y densidad de población, especialización del trabajo, tributos, monumentalidad, edificaciones públicas, burocracia, escritura y ciencia, desarrollo del comercio exterior y la existencia de una organización estatal basada en la residencia más que en el parentesco.

2 En adelante BA.

3 Cf. Childe, 1950; Storey, 2006; Yoffee, 2005. 
o la aparición de funcionarios, junto a una reducción del ámbito social en el que actúa el parentesco (Milevski, 2010: 164; Jaruf, 2019: 9). Estos elementos permiten el crecimiento de los asentamientos -en población y tamaño- junto a un aumento de la producción de determinados bienes.

Existe cierto acuerdo de que el proceso de urbanización en el Levante inició a partir del BA IB (3500/3400-3000 a.C.). ${ }^{4}$ Previamente, durante el BA IA (3700$3500 / 3400$ a.C.), la sociedad levantina se caracterizaba por mantener una vida rural, aunque con cierta jerarquía de asentamientos. Posteriormente, durante el BA IB, cambia la fisonomía de estos asentamientos, evidenciándose en algunos sitios la aparición de fortificaciones con torres que encerraban grandes edificios públicos, almacenes y talleres especializados en la producción masiva de ciertos bienes como pan, metales o aceite de oliva (Jaruf, 2019: 17; de Miroschedji, 2009: 105). Al mismo tiempo, se desarrollaron redes de interacciones regionales e interregionales con comunidades foráneas como Egipto, quien se constituiría como centro en el marco de estas relaciones de carácter centro-periféricas (cf. Campagno, 2010). Esta nueva red de asentamientos fortificados en el sur del Levante durante el BA II habría presentado rasgos comunes: ubicación en terrenos elevados, en donde se habrían redefinido identidades locales y acumulación de bienes de prestigio por partes de líderes que podrían haber necesitado demostrar su poder al interior de los muros, o hacia las poblaciones exteriores a los mismos; simplificación de la cultura material por el desarrollo de industrias regionales especializadas y de redes de distribución de bienes; y ausencia de expresión clara de status (Greenberg, 2019: 95).

El proceso de urbanización se acentúa durante el BA II-III (3000-2300 a.C.), cuando se documenta el abandono de antiguos asentamientos del BA I, y la fundación de nuevos sitios de mayor tamaño fortificados, junto a algunos casos de desarrollos desde aldeas del BA I. Según de Miroschedji (2009: 106), existieron diferentes tipos de asentamientos: transitorios, pequeñas aldeas, grandes aldeas, urbes fortificadas y grandes asentamientos fortificados, entre los que destacan Ai (11 ha), Tel Yarmuth (16 ha), Beth Yerah (25 ha), entre otros.

Las principales hipótesis ${ }^{5}$ respecto al origen de urbanismo levantino destacan factores como la migración de poblaciones foráneas (por ej., Kenyon, 1960), el difusionismo cultural (por ej., Kempinski, 1978), la emulación por contacto (por ej., van den Brink y Levy, 2002), pero sobre todo causas endógenas (por ej., de Miroschedji, 1971; Milevski, 2016). A pesar de sus diferencias, estas teorías comparten el hecho de no considerar de manera suficiente a las poblaciones móviles de las regiones áridas y semiáridas, a las cuales sólo se les concede un rol marginal en este proceso. Estas poblaciones no suelen ser tenidas en cuenta por los estudios sobre el urbanismo en la antigüedad o, a lo sumo, se les concede

4 Si bien tradicionalmente se ha pensado que las primeras ciudades emergieron en la Baja Mesopotamia y que luego se difundió el fenómeno urbano hacia regiones vecinas, recientemente, a partir de los nuevos hallazgos arqueológicos se ha cuestionado la idea de que áreas como el Levante hayan constituido una "segunda urbanización" (cf. Jaruf, 2019).

5 Para un desarrollo de las hipótesis sobre el origen del urbanismo en el sur del Levante cf. Jaruf, 2019. 
un rol subsidiario, ya que se solía asumir que los pastores y los asentados eran mutuamente excluyentes (Porter, 2012).

Los pastores móviles del sur del Levante habitaron las áreas marginales a las zonas nucleares en donde se llevó a cabo el proceso de urbanización. Con base en las similitudes en la cultura material que presentan los yacimientos ubicados en las regiones áridas, a su economía de tipo pastoril y a su modo de vida móvil, se los ha denominado cultura Timnita ${ }^{6}$ (Rosen, 2017). Recientemente, se ha comenzado a valorizar su participación en redes de intercambios con estos primeros asentamientos urbanos, y se ha propuesto que los pastores proveyeron de bienes de diversa índole a las urbes levantinas (por ej. Finkelstein, 1995; Abe, 2008; Abu-Azizeh, 2013; Rosen, 2017). Es por este motivo que consideramos relevante repensar la urbanización en el sur del Levante a partir del estudio de estos pueblos pastoriles, los cuales pudieron haber tenido un rol importante en este proceso.

La forma de subsistencia timnita, el pastoralismo, puede definirse como el modo de vida en el cual la práctica del pastoreo y de manejo de rebaños de animales domésticos es la actividad económica principal (Khazanov, 1984; Potts, 2014). Las comunidades que participan en estos modos de producción suelen tener altos grados de circulación entre diferentes nichos ecológicos, ya que las necesidades de pasturas hacen necesario el aprovechamiento estacional de diferentes áreas geográficas. De esta manera, se han planteado varias formas de movilidad de los pastores: nomadismo (movilidad de todos los integrantes y ausencia de agricultura), seminomadismo (movilidad con muchas residencias), semisedentarismo (actividad pastoril durante una parte del año) y trashumancia (ganado de poblaciones sedentarias movilizado por sólo una parte de la comunidad) (Digard, 1982: 13). Sin embargo, en este trabajo preferimos utilizar el término "móvil" para referirnos a las poblaciones no-sedentarias, ya que de esta manera evitamos la utilización de estas subcategorías, siempre arbitrarias. Al mismo tiempo, con este concepto contemplamos la diversidad de grados de circulación que pudiesen haberse dado en esta población, y que en el registro arqueológico es difícil de determinar. De esta manera, podemos definir la movilidad como la capacidad o necesidad de trasladado de un lugar a otro de personas o grupos que realizan actividades que dependen de cierto grado de movimiento (Wendrich y Barnard, 2008: 6).

La economía de las poblaciones pastoriles se basaba no sólo en la cría de ganado, sino también en sistemas de múltiples recursos y estrategias de subsistencia, especialmente a partir de la explotación de sus productos secundarios, ${ }^{7}$ el caravaneo, la metalurgia, etc. (por ej. Honeychurch y Makarewicz, 2016; Rosen, 2017). Asimismo, la producción pastoril y la movilidad implican a su vez la generación de un repertorio tecnológico, social y simbólico, que resultan en

6 La cultura o complejo Timnita fue definido por primera vez por Kozloff en 1973 basándose en investigaciones realizadas en el curso de estudios y excavaciones en Sinaí y el Néguev por Rothenberg, y lleva el nombre del valle de Timna, en el sur del Néguev.

7 Los productos secundarios consisten en bienes obtenidos a partir de la producción animal: por ej. lana, leche, etc. 
un modo de vida y una ideología específica, donde es central la co-comunidad entre animales y humanos (Salzman, 2004: 142; Honeychurch, 2015).

\begin{tabular}{llll} 
Años a.C. Cal. & Levante meridional & Néguev y Sinaí \\
\hline $4300-3800 / 3700$ & Calcolítico Tardío & Timnita Medio \\
\hline $3700-3500 / 3400$ & Bronce Antiguo IA & \\
$3500 / 3400-3000$ & Bronce Antiguo IB & \\
$3000-2800$ & Bronce Antiguo II & Timnita Tardío
\end{tabular}

Cuadro cronológico comparativo entre las regiones del Néguev y del Sinaí y del Levante meridional (tomado y modificado de Rosen, 2017 y Daizo, 2019).

\section{El proceso de urbanización y su periferia}

Como dijimos con anterioridad, algunos investigadores han comenzado a indagar sobre las vinculaciones entre el pastoralismo móvil y el proceso de urbanización (Abe, 2008; Anfinset, 2010; Porter, 2012; Rosen, 2017). Se ha destacado que estas poblaciones se vieron influenciadas de diferentes maneras por los cambios acontecidos en los asentamientos agropastoriles de la región del sur del Levante, especialmente a través del intercambio de bienes y servicios. Esto se observa en el registro material, el cual presenta cambios con respecto al período previo (Calcolítico).

Según Levy (1992), durante el Calcolítico se produjeron cambios en la producción pastoril, debido al incremento de la población del sur palestino, que supuso la colonización del interior del norte del Néguev. Esto, al mismo tiempo que la introducción de cultivo en los principales valles interiores, proporcionó el catalizador necesario para la transformación del pastoreo basado en la cercanía a aldeas, a un sistema de trashumancia completo. Dichos cambios se evidenciaron en un mayor control de rebaños de ovejas y cabras, junto a la intensificación de la explotación de productos secundarios. La transición del período Calcolítico al BA estuvo marcada por el colapso del sistema de asentamientos en el valle de Beersheva. Puede ser que este cambio se haya debido a las modificaciones en el modo de producción pastoril durante BA I (Levy, 1992: 74).

En este período también tuvo lugar el pasaje de un sistema productivo de pastoreorecolección (Timna Medio), basado en la caza-recolección con incorporación de animales domésticos, a un pastoralismo móvil (Timna Tardío), en donde se estableció un sistema económico complejo de relaciones entre grupos pastoriles del desierto y el sistema urbano, junto a sus aldeas satélites (Rosen, 2017). Con este sistema se establecieron nuevos patrones demográficos en los asentamientos semi temporarios del desierto y una dinámica de intercambio interpretada en los términos del modelo de centro-periferia (Rosen, 2017: 167). Asimismo, esta transición está relacionada con la emergencia de la demanda de cobre por los asentamientos calcolíticos a finales del V milenio a.C. De esta manera, Rosen (2017: 168) afirma que durante el período Timna Tardío, la aparente expansión del pastoralismo de subsistencia estaba vinculada al surgimiento de mercados urbanos. 
Durante el BA I se disuelven algunas aldeas e incrementa la actividad de los pastores móviles en las regiones áridas, al mismo tiempo que se intensifican los intercambios con Egipto (Anfinset, 2010: 55). Según Anfinset (2010), la periferia jugó un rol importante en la formación de las primeras ciudades y del Estado egipcio, en la medida en que estos dominaron más territorios y comenzaron a controlar las redes de intercambios que atravesaban el desierto. En este sentido, el incremento de la producción pastoril proveyó las bases de la transformación social del sur del Levante (Anfinset, 2010: 184). Por este motivo, Anfinset (2010: 200) considera al pastoralismo nómada como un actor dinámico de difusión y cambio cultural entre diferentes modos de subsistencia.

Otros investigadores también afirman que las comunidades de pastores móviles habrían sido de vital importancia para el proceso de urbanización debido a que abastecieron a los núcleos urbanos de bienes provenientes de regiones áridas (por ej. Abe, 2008; Rosen, 2017). Esto repercutió en el modo de vida y en la economía de los pastores, impulsando un incremento y una especialización de las relaciones de intercambios y de la producción de herramientas líticas (Abe, 2008). El surgimiento del pastoralismo móvil sobre la base de la intensificación de la cría de caprinos y la incorporación de mayores áreas de explotación pastoril, y de productos secundarios, ha sido considerado como una respuesta al surgimiento de la demanda de los mercados urbanos (Zeder, 1991; Levy, 1992).

Junto a los productos derivados del pastoralismo, también se evidencia la circulación de bienes elaborados a partir de la extracción de minerales como pedernal eocénico proveniente de la cuenca de Jarf, materiales de arenisca del Néguev central y cobre del sur del Sinaí, los cuales habrían sido enviados hacia los recientes asentamientos urbanizados (cf. Abe, 2008). A juzgar por estos testimonios de circulación de bienes, y su contemporaneidad con la aparición de las urbes levantinas, el proceso de urbanización probablemente haya tenido influencia en que las poblaciones pastoriles de las regiones áridas se involucraran en mayor medida en los sistemas de intercambio, y orientaran sus actividades productivas al mercado urbano. Asimismo, este proceso habría hecho más dependientes a estos grupos de los productos urbanos, como alimentos agrícolas, telas, etc. (Abe, 2008: 537-538).

A diferencia de las propuestas precedentes, algunos autores criticaron la idea de la existencia de un sistema pastoril especializado en este período. Arbuckle y Hammer (2018) afirman que las evidencias son escasas, ya que argumentos como el de Rosen se basan principalmente en que hay un aumento en el número de campamentos documentados en el Néguev, la presencia de corrales para ganado y capas de excrementos en refugios de rocas como Ramon, que supuso representaban un "emplazamiento intensivo" (Rosen, 2017: 173). Sin embargo, la falta de evidencia directa de estacionalidad y movilidad, las reconstrucciones de subsistencia limitadas y la presencia de sitios residenciales a un día de caminata de fuentes de materias primas, plantean serias dudas sobre el carácter especializado, nómada e incluso pastoril de esta cultura (Arbuckle y Hammer, 2018: 24). Asimismo, estos autores proponen concebir el pastoralismo del suroeste asiático como un conjunto de adaptaciones dinámicas y extensas que respondieron a nivel local, regional e incluso suprarregional a los cambios en la economía, la ecología, la política y la demografía (Arbuckle y Hammer, 2018: 43). 
Como se puede apreciar, varios autores consideran que la presencia de grupos pastoriles móviles en las regiones áridas periféricas o marginales pudo haber jugado algún rol en el proceso de urbanización durante el BA. Sin embargo, no parece haber común acuerdo en el carácter de esta participación. Por un lado, algunos investigadores consideran que el proceso de urbanización, especialmente el incremento de la demanda de los sectores urbanos, modificó las prácticas pastoriles de las poblaciones móviles, quienes se adaptaron para extraer materias primas de regiones áridas e incrementar su producción pastoril, adecuándose de esta manera a los cambios económicos de la región (Zeder, 1991; Levy, 1992; Rosen, 2017). También se planteó que, si bien estos grupos crecieron demográficamente e intensificaron su producción, fue necesario el acceso a recursos lejanos y redes de intercambio para el proceso de urbanización (Abe, 2008). Por otro lado, se ha estimado al pastoralismo móvil como un factor dinámico de cambio cultural, que pudo provocar modificaciones en las sociedades sedentarias (Anfinset, 2010). Sin embargo, atento a los problemas que presenta determinar las prácticas sociales pastoriles a través del registro arqueológico, algunos autores afirman que no se puede establecer esta relación por el carácter escaso de las evidencias en el sur del Levante (cf. Arbuckle y Hammer, 2018).

En definitiva, el incremento de la producción pastoril, de los intercambios y de las poblaciones de pastores móviles parece estar fuertemente relacionado con el fenómeno urbano. Nuestra hipótesis es que los pastores móviles habrían tenido un rol fundamental en este proceso, ya que no sólo proveyeron de los bienes necesarios para los asentamientos urbanos, sino que su presencia, la cual posiblemente haya variado entre pacífica y hostil, pudo ser un factor más que influenció en el amurallamiento y la concentración de las nacientes urbes levantinas. Si bien es difícil demostrar esto, a partir del análisis de estas poblaciones, creemos que podemos aproximarnos a formular algunas consideraciones relevantes para el estudio de la problemática del proceso de urbanización en el sur del Levante. 


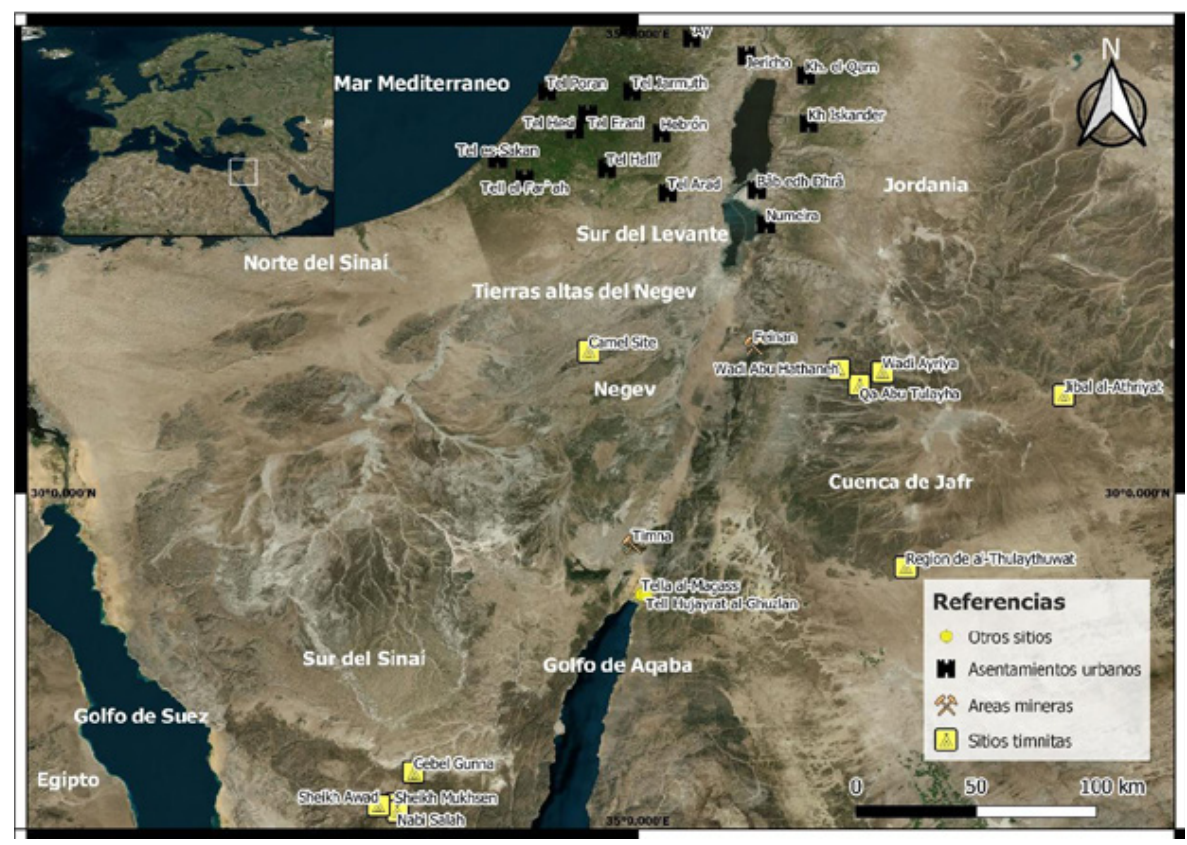

SEQ llustración \* ARABIC 1 Mapa del sur del Levante y del Sinaí con los sitios mencionados en el artículo (elaborado con QGIS).

\section{Los pastores móviles del desierto durante el proceso de urbanización}

Durante el Timnita Tardío, se observa una expansión demográfica (más cantidad de sitios estacionales y mayor área de distribución de estos) y la existencia de prácticas económicas de subsistencia con base en el pastoreo de cabras y ovejas y de cacería. Asimismo, se documenta agricultura en microambientes e importación de granos, producción a nivel doméstico y producción sistemática de ciertos bienes con destino a los mercados urbanos, por lo que se supone que hubo un mayor impacto de la influencia de las sociedades urbanas en estas regiones (Rosen, 2017: 169).

La arquitectura timnita consistía en asentamientos semipermanentes de recintos cercados (probablemente para alojar los rebaños) con cuartos adjuntos, santuarios en el desierto con un simbolismo cosmológico particular, y enterramientos en túmulos ${ }^{8}$ y nawamis ${ }^{9}$ asociados a esos santuarios. Entre su cultura material destaca una industria lítica propia (puntas de flechas de piedra, taladros microlíticos, etc.), herramientas de cobre, cuentas de conchas marinas

8 Los túmulos consisten en una acumulación de piedras con base circular con entierros en cista en su núcleo, cercanos a sitios habitados. En la mayoría de los casos no se encontró un ajuar asociado.

9 Los nawamis consisten en estructuras cilíndricas, de aproximadamente 3 a $7 \mathrm{~m}$ de diámetro externo y $2 \mathrm{~m}$ de alto, abovedado con arcos de corbel y estaban destinados a entierros múltiples en el centro/sur del Sinaí. 
y cerámica que aparece tardíamente. Se determinó que su distribución geográfica abarcó el sur del Sinaí, sur de Jordania, noroeste de Arabia y el Néguev (cf. Rosen, 2017).

Durante el período Timnita Medio, la economía se había basado en un sistema de pastoreo-recolección, con patrones de asentamientos determinados por la movilidad pastoril estacional y conectados a sistemas de intercambios relacionados con la incipiente producción de cobre para transportar a sitios sedentarios (cf. Abe, 2008). En cambio, a partir del Timnita Tardío, estas poblaciones modificaron su modo de subsistencia, caracterizándose en este momento por un sistema pastoril móvil intensivo de aprovechamiento de múltiples recursos (Rosen, 2017). Según Rosen (2017), la producción en este momento estaba orientada a la exportación de ciertos bienes para suplir las demandas de los mercados urbanos, especialmente de cobre, como así también de productos secundarios pastoriles, raspadores tabulares, etc. De esta manera, se evidencia un mayor contacto con las poblaciones urbanizadas del sur del Levante, al mismo tiempo que con el Estado egipcio, junto a un crecimiento demográfico y de asentamiento temporarios. Finalmente, durante el periodo Timnita Terminal (BA III), colapsa el complejo pastoral Timnita del Néguev central, aparentemente producto de la caída de los mercados urbanos, como el de Arad, y de la mayor presencia egipcia en el Sinaí (Rosen, 2017).

En los inicios del BA no sólo se desarrolla el fenómeno de la urbanización en el Levante meridional, sino que también acontece el surgimiento de dinámicas estatales expansivas en Egipto, quien interactuó con las urbes levantinas y los grupos pastoriles de las regiones intermedias. Éstas se habrían materializado en sitios como Tel Sakan o Ein Besor, al sur del Levante, y habría implicado el establecimiento de relaciones asimétricas, particularmente por la búsqueda de bienes exóticos por parte del dispositivo estatal en el Levante, convirtiéndolo de esta manera en su periferia (cf. Campagno, 2010). Durante el BA Ib-II se repliega la presencia egipcia en el Levante, al mismo tiempo que sitios urbanos, tales como Tel Arad, Tel Erani, etc., comienzan a presentar mayores niveles de concentración, edificios públicos y murallas. Esto pudo haberse dado tanto por el establecimiento de una ruta marítima entre Egipto y Biblos, o porque, a medida que fueron emergiendo nuevas organizaciones políticas sudlevantinas, Egipto encontró socios comerciales locales que se encargarían directamente del envío de productos al valle del Nilo (Campagno, 2010: 203-204).

La participación de las poblaciones timnitas en las redes de intercambio a larga distancia se evidencia a partir de los sitios asociados a esta cultura material, en donde se hallaron diversos artefactos provenientes del valle del Nilo, del Levante meridional y de regiones lejanas como Anatolia. No es sencillo determinar la manera en que se llevó a cabo la circulación de bienes. Probablemente tomó diferentes formas a lo largo del tiempo, pudiendo en determinado momento haber sido activa, es decir, que estas comunidades habrían sido las encargadas de llevar a cabo estos intercambios, y en otros momentos, haber participado de manera subsidiaria o secundaria.

A los efectos de determinar la influencia del proceso de urbanización en los cambios acaecidos en estas comunidades pastoriles, a continuación, analizaremos 
algunos de sus aspectos arquitectónicos y demográficos, junto a los objetos destinados a los intercambios hallados en las regiones del Néguev, sur del Sinaí y sur de Jordania. Además, revisaremos la hipótesis de que la urbanización del asentamiento Tel Arad pudo haber sido llevado a cabo por estas poblaciones móviles.

\section{Arquitectura y demografía}

A partir del Timnita Tardío, se observa un incremento de asentamientos a lo largo del desierto del Néguev central y centro-oeste. Estos consistían en recintos cercados con cuartos adjuntos (Rosen, 2017: 169-170). Algunos presentan cierta influencia de Arad en su arquitectura, evidenciado en las estructuras de cuartos amplios rectangulares con bancos internos y pilares (ver más abajo). Según Rosen (2017: 171) estos se diferencian de los de Arad en el hecho de que habrían funcionado como recintos o rediles con muros de $50 \mathrm{~cm}$ de alto para guardar ganado, mientras que los de Arad habrían tenido grandes muros, aparentemente para funcionar como un espacio privado dentro de la urbe.

En las tierras altas al oeste del Néguev se registraron 200 sitios de tres tipos: permanentes, temporales y campos de mojones (Saidel y Haiman, 2014: 171). Se estimó que la población total de esta región pudo haber sido de 800 personas, mientras que los asentamientos temporales de entre 60 y 120 (Saidel y Haiman, 2014: 172). Estos pobladores pudieron haber transitado la región y aprovechado los asentamientos estacionales como puntos de descanso o de pastoreo estacional.

La ocupación de algunos sitios del Néguev central data del mismo período (BA IB- II) en que se desarrolla el proceso de urbanización y comienzan a vislumbrarse estructuras defensivas (murallas y torres) en varios sitios levantinos. De esta manera, se evidencia que el fenómeno urbano convivió con el surgimiento de emplazamientos semipermanentes en el desierto.

Tradicionalmente se sugirió que en las tierras altas del Néguev la primera ola de ocupación habría ocurrido entre el BA II y el BA III, cuando deja de haber evidencia de ocupación en estos sitios y en Arad (cf. Rosen, 2017). Sin embargo, a partir de nuevas dataciones, se ha cuestionado la hipótesis del abandono de estos sitios durante el BA III. Esto se debe a que se encontraron objetos que datan de este período en Arad (especialmente conchas marinas) y que la actividad minera del valle de Aravá continuó durante el BA III, la cual pudo haber sido llevada a cabo por los pastores del Néguev o los habitantes de Arad (Finkelstein et al., 2018).

Algunos sitios del sur del Sinaí fueron definidos como asentamientos asociados a una economía pastoril, al mismo tiempo que a la producción y exportación de cobre al Levante y Egipto. Sitios como Sheikh Muksen, Nebi Salah y Sheikh Awad presentan cierta influencia arquitectónica de Arad, por lo que se interpretó como una presencia efectiva de Arad en el sur del Sinaí. Según algunos autores podrían haber sido colonias o puestos de avanzada (por ej. Amiran et al., 1973). En cambio, otros los consideran puestos comerciales intermediarios entre Arad y las rutas de cobre (por ej., de Miroschedji, 1986; Beit-Arieh, 
2003). En este sentido, se discute si la extracción y exportación de cobre fue llevada a cabo por población local, por levantinos que colonizaron la región o incluso, como veremos luego, si los habitantes de esta región intervinieron en la fundación de Arad. Otros sitios poseían recintos ovales de varios tamaños, orientada de este a oeste, con patios semicerrados. Estas contenían diversas habitaciones y estructuras de almacenamientos, en donde se hallaron puntas de flecha transversales, tiestos y raspadores con forma de abanico.

A partir del tercer milenio a.C., comienzan a realizarse expediciones egipcias al sur del Sinaí, y se observa cierta presencia efectiva impuesta por medio de la violencia. Esto se evidencia a partir de bajorrelieves ${ }^{10}$ en Wadi el Hummur durante el reinado de Den. Estas inscripciones conmemoran la victoria egipcia sobre la población local, denominada Iuntiu, ${ }^{11}$ y la apropiación del cobre y la turquesa local (Tallet, 2010).

Se ha documentado la presencia de poblaciones pastoriles desde el Calcolítico en la cuenca de Jarf, sur de Jordania (cf. Abe, 2008). En esta región se hallaron varias habitaciones agrupadas, con cercados que se asemejan a las que utilizan grupos seminómadas en la actualidad como corrales para animales. Se sugiere que estas estructuras fueron pequeños campamentos pastoriles ocupados estacionalmente, debido a la poca abundancia de artefactos y a que los sedimentos son poco profundos (Abe, 2008: 523).

Otros sitios pastoriles relevantes se han registrado en al-Thulaythuwat. En la región circundante se encontraron varios campamentos estacionales, probablemente utilizados temporalmente por pastores móviles, caracterizados por tener un tamaño relativamente pequeño, cerramientos con piedras y una pequeña cantidad de herramientas de pedernal (Abu-Azizeh, 2013: 153). Los tamaños reducidos de estos asentamientos podrían indicar la ocupación por pocos grupos de pastores, probablemente unidades familiares o households.

Además de estos campamentos estacionales, Abu-Azizeh ha identificado otros de ocupación más prolongada. Estos poseen una organización más compleja de las unidades formadas por círculos de piedra, las cuales guardan cierta semejanza con las del BA II del Sinaí, y mayor cantidad y variabilidad en los tipos de herramientas de pedernal (Abu-Azizeh, 2013: 155). A esta jerarquía de sitios de distintas estacionalidades de ocupación, similar a la que se observa en el Néguev, se le suman otros que poseen patios cerrados, lo cual podría suponerse que eran utilizados, como en otras regiones, para la cría de ganado.

10 El tema de estos tres bajorrelieves consiste en la representación del rey Den, con la corona blanca y en ocasiones roja, masacrando con una maza a los pobladores locales. Den está seguido por un séquito de soldados y portasandalias, al mismo tiempo que acompañado por deidades como una figura antropomorfa con tocado emplumado y la insignia de la divinidad canina Upuaut (Tallet, 2010: 99).

11 El nombre de luntiu, o en ocasiones Mentiu, aparece en algunas fuentes egipcias para referirse a ciertas poblaciones que habrían habitado el sur del Sinaí, y que solían ser conquistadas por los reyes egipcios. Se han registrado al menos diez apariciones en documentos del Reino Antiguo, entre ellas podemos mencionar el bajorrelieve de Keops en Wadi Maghara (Sinaî), en donde aparecen masacrados los luntiu (Tallet, 2010: 102). 


\section{Bienes de intercambio}

En uno de los principales asentamientos temporales del Néguev central, Camel Site (3000-2700 a.C.), se registraron tanto objetos líticos (lascas, herramientas de moliendas, herramientas ad-hoc, etc.), como así también bienes provenientes de regiones lejanas: cáscaras de huevos de avestruz, conchas marinas, hematita trabajada, herramientas de cobre, obsidiana y cerámica (Rosen, 2003). Respecto a las puntas de flechas transversales y lunares que se hallaron en el sitio, Rosen (2003: 755) afirma que pudieron haber servido para la caza e incluso para bajos niveles de guerra.

En Camel Site se documentaron además objetos provenientes del valle del Nilo, entre ellos madreperla nilótica, cuentas de oro y gran cantidad de cuentas de conchas marinas provenientes del mar Mediterráneo y del mar Rojo. Asimismo, la metalurgia, el uso de cobre puro y arsenical, testimonia la participación de estas poblaciones en el tráfico de metales. La presencia de obsidiana proveniente de Anatolia, y cuarzo del sur del Néguev o del Sinaí, indica que sus pobladores intervinieron en un sistema de intercambios de larga distancia, e incluso podrían haber mantenido intercambios con Arad a cambio de granos.

En el sur del Sinaí la actividad económica de subsistencia habría sido la cría de cabras, junto a la caza de gacelas y cabras montañesas salvajes. Si bien no se encuentran artefactos que indiquen prácticas agrícolas, la presencia de pithoi y holemouth jars sugiere la posibilidad de importación y almacenamiento de granos desde asentamientos agrícolas. Muchas de estas vasijas tenían forma globular con paredes delgadas, característica compartida con las documentadas en Arad del BA I-II. Respecto a las jarras con cuello, sólo su forma es comparable a las realizadas en Arad, mientras que el tratamiento de superficie y las arcillas parecerían tener un origen relacionado con Egipto (Bar-Yosef et al., 1986: 149).

Teniendo en cuenta que se practicó la agricultura intensiva en Arad, asentamiento más cercano al desierto, sumado al desarrollo del transporte con burros, y la presencia de herramientas de molienda y recipientes de almacenamiento en todos los sitios, se puede pensar en prácticas de intercambio de productos locales por granos y alimentos de otros sitios (Beit-Arieh, 2003; Rosen, 2017: 175). A cambio, estas poblaciones habrían extraído, pulido y exportado turquesas y cobre para enviarlos a los asentamientos levantinos y al valle del Nilo (Beit-Arieh, 2003: 439).

En el área de Gebel Gunna se documentaron doce nawamis dispersos cercanos a tres sitios de habitación (Bar-Yosef et al., 1986). El ajuar que se encontró en estos nawamis consistía en huesos humanos, objetos de cobre y de pedernal, un hacha de basalto pulido y raspadores en forma de abanico (Bar-Yosef et al., 1986: 123-126). Asimismo, se registraron algunos ítems ornamentales como cuentas, colgantes y brazaletes. La mayoría de los moluscos hallados en los nawamis provenían del mar Rojo, y con estos se elaboraron algunas de las cuentas, mientras que otras fueron realizadas con turquesas. Los objetos de cobre contenían una composición similar a los que se hallaron en otros sitios como 'Ein Huderah y Sherkh Awad (Bar-Yosef et al., 1986: 146). En total, se encontraron 26 individuos inhumados de diferentes edades. En uno de los 
nawamis se hallaron 3 individuos con la misma enfermedad congénita, coxa vara, por lo que se ha supuesto la posibilidad de reutilización de los enterramientos por una misma familia (Bar-Yosef et al., 1986: 163). En definitiva, los bienes hallados en estos sitios provenían de regiones diversas, por lo que se podría pensar que los habitantes del Sinaí estaban integrados a una extensa red de circulación de bienes interregional.

Al norte de la península del Sinaí se hallaron asentamientos con bienes foráneos (egipcios y levantinos), lo que podría indicar que en el norte era por donde pasaban algunas de las rutas de intercambio entre Egipto y el Levante. Asimismo, en dichos sitios se documentó mayor cantidad de cerámica egipcia que levantina (en proporción de 4:1), entendiéndose esto como una preponderancia egipcia en los intercambios (Oren, 1986).

Entre los sitios más característicos de Jordania podemos mencionar Wadi Abu Yulayha ocupado desde el Neolítico Precerámico B hasta el BA. Se determinó que este sitio era un centro de producción de raspadores tabulares (Abe, 2008: 198). En Qa' Abu Tulayha se encontraron ocho áreas de extracción asociadas a la concentración de núcleos con los que se habrían producido 15.000 raspadores, al mismo tiempo que en Jibal al-Athriyat se encontraron 80 sitios de extracción y pulido de nódulos de pedernal (Abu-Azizeh, 2013: 162).

Según Abe (2008), los sitios de la cuenca de Jarf eran centros de producción de raspadores tabulares, posiblemente para usos rituales e incluso destinados al intercambio con otras poblaciones. La producción de estos líticos está asociada a la cultura Timnita, lo que podría dar cuenta de una esfera de interacción cultural pastoril entre el Sinaí, Néguev y Jordania. De esta manera, los asentamientos podrían haber sido ocupados por poblaciones pastoriles móviles, ya que no se evidenció la existencia de agricultura en la región, además de ser un área en la que se suele practicar el pastoreo móvil incluso en la actualidad (Abe, 2008).

Abe (2008) afirma que el proceso de urbanización provocó el incremento de la producción de herramientas líticas en la cuenca de Jarf. Teniendo en cuenta la especialización productiva de varias regiones para exportar productos a áreas urbanizadas, este mismo autor (Abe, 2008: 535) sugiere que los grupos móviles también participaron incrementando la producción de herramientas líticas de pedernal eocénico extraído del lugar, para ser utilizado por poblaciones sedentarias. Desde el período Calcolítico se documentan raspadores tabulares en Teleilat Ghassul, siendo aparentemente los sitios ubicados en la zona de Aqaba, Tell al-Magass y Tell Hujayrat al-Ghuzlan, los principales centros redistributivos de estos objetos, conectando así la producción jordana de herramientas con quienes los utilizaban en el sur del Levante (Abu-Azizeh, 2013: 168). Según Abe (2008: 536), con el desarrollo del urbanismo se verifica también un incremento de la producción de raspadores tabulares, por lo que concluye que la producción en sitios pastoriles se incrementa y se especializa en materias primas, como consecuencia del proceso de urbanización durante el BA.

Es interesante destacar que hacia fines del cuarto milenio a.C., en el sur del Levante se observa una disminución notable de la presencia de puntas de flechas. Mientras que en la mayoría de los sitios sedentarios casi desaparece su 
presencia, en las regiones áridas esta se mantiene estable. Generalmente, el uso de puntas de flecha está reducido a dos actividades: la caza y la guerra. Probablemente su función haya sido la primera, ya que las actividades pastoriles necesitan de una economía complementaria, tanto de contactos con sociedades agrícolas, como así también de productos obtenidos a través de la cacería, lo cual se evidencia en la presencia de "cometas del desierto". ${ }^{12}$ Sin embargo, también se podría considerar la posibilidad de que esos grupos móviles hayan mantenido conflictos con otras poblaciones o entre sí ${ }^{13}$ debido a su modo de vida móvil, y al uso de las rutas de intercambios, en las que inicialmente podrían haber ponderado al menos hasta que los centros estatales y urbanos comenzarían a imponer su hegemonía.

Por último, en la región de Feinan, una de las fuentes de cobre durante el período Calcolítico y el BA, se evidenció la presencia de recintos de piedra y herramientas de pedernal típicos de la cultura Timnita. Esto podría dar cuenta de la asociación de grupos pastores móviles con la temprana extracción y circulación de cobre en esta región (Abu-Azizeh, 2013: 165).

\section{Tel Arad, ¿urbanización pastoril?}

Por último, algunos autores han propuesto que la urbanización de Tel Arad se habría dado a partir de un fenómeno desarrollado en el Néguev y el Sinaí (Finkelstein, 1990). De esta manera, el desarrollo del asentamiento urbano de Arad habría sido consecuencia de la sedentarización de ciertos grupos móviles pastoriles, los que utilizaron dicho sitio como base de operaciones para los intercambios de productos provenientes de las regiones áridas hacia las urbes levantinas.

Tel Arad se encuentra ubicado al norte del Néguev en una región semiárida sin suministro estable de agua. Su fundación data aproximadamente del Calcolítico, en donde se registra la existencia de un asentamiento grande o aldeas dispersas pequeñas con una cultura material similar a la de Beersheva. Durante el BA II se evidencia la construcción de un nuevo asentamiento más grande rodeado con muros defensivos de piedra de $2,4 \mathrm{~m}$, junto a torres semicirculares y compuesto de calles conectadas con patios abiertos y grupos separados de unidades de vivienda (Finkelstein, 1990: 35). En este período se documenta el abandono de sitios egipcios en el sur del Levante, al mismo tiempo que se expanden asentamientos semitemporarios en el Néguev y el Sinaí con una cultura material similar a la de Arad (Greenberg, 2019: 76).

La estructura urbana de este sitio consistía en una distribución ordenada de calles concéntricas y radiales, con una zona donde se ha supuesto que se

12 Los cometas del desierto eran estructuras con forma de " $\mathrm{V}$ " que funcionaban como trampas para la caza de fauna salvaje, como las gacelas.

13 Se considera que los nómadas tenían un aspecto bélico debido a que estos grupos se constituían en una fuerza paramilitar en permanente movilización parcial, que se podría convertir en un activo militar importante para los Estados, u organizaciones políticas que lo pudieran capitalizar (Rowton, 1982). 
encontraban emplazados un templo, un palacio ${ }^{14}$ y un depósito. Además, se registraron estructuras aparentemente administrativas y una plaza de mercado. Uno de los elementos más característicos de este asentamiento urbano es la arquitectura de las unidades de vivienda denominada "casa aradiana". Éstas consistían en habitaciones rectangulares, con bancos a lo largo de las paredes y uno o dos postes en el centro para sostener el techo. A su vez, poseían más de una habitación, una principal de mayor tamaño, y tenían patios con instalaciones rodeadas por un muro que las separaba de la calle y de otras casas, generando así un lugar cerrado (Finkelstein, 1990: 36). Todos los habitáculos eran accesibles únicamente desde el patio, y en el principal se documentan instalaciones de cocina y de almacenamiento (Greenberg, 2019: 84). Es interesante mencionar que se encontraron sitios en el sur del Sinaí que guardan cierta relación con la estructura de estas unidades domésticas de Arad, como ya se mencionó anteriormente.

Si bien en un principio, como ya mencionamos, la similitud arquitectónica que guardaban los sitios del sur del Sinaí con los de Arad fue interpretada como una posible colonización u ocupación de levantinos en la región del Sinaí, Finkelstein (1990: 39) sugiere que los habitantes de estas regiones fueron parte de las poblaciones móviles que se asentaron y fundaron Arad. Una de las razones podría haber sido la aparición de oportunidades de intercambio con las nacientes urbes levantinas, $o$ incluso la necesidad de tierras de cultivo accesibles a la región de Arad. De esta manera, mientras algunos segmentos o parcialidades de poblaciones del desierto se asentaron en Arad, otros podrían haber mantenido las prácticas pastoriles móviles y de caravaneo por las regiones áridas.

Existe la posibilidad de que Arad haya sido un asentamiento urbano caracterizado por ser un centro de intercambios de las poblaciones del sur. Es decir, pudo haber funcionado como un puesto de intercambios protegido, en donde diferentes poblaciones dedicadas al caravaneo, extracción de materias primas y pastoreo llevaban sus productos, para luego intercambiarlos con el resto de los asentamientos levantinos. Sus habitantes podrían haber adquirido materias primas para la producción de artesanías (cerámica, textiles, cuentas de piedras o conchas marinas) o para su tráfico. También se ha sugerido que era un centro intermediario en la distribución de bitumen del mar Muerto y cobre de Feinan, ambos registrados también en Egipto. Esto sumado a las conexiones con el Néguev y el Sinaí, podría dar cuenta de que Arad era un nodo de intercambio importante en el BA (Greenberg, 2019: 82).

Finkelstein (1990: 43) afirma que la arquitectura de Arad podría dar cuenta de un pasado pastoril móvil de las poblaciones asentadas, ya que ésta poseía patios separados entre sí con espacios abiertos (típicos entre pastores móviles asentados), y las salas con pilares centrales y patios rodeados con muro se asemejan a las tiendas de beduinos. Si tenemos en cuenta que los pastores del desierto

14 Se ha discutido la categoría de templo y de palacio para describir a estas estructuras de gran tamaño, ya que en realidad podrían haber sido grandes households de ciertos líderes (Greenberg, 2019: 80). 
pudieron haber tenido un rol fundamental en la circulación de las redes de intercambio y que Arad tenía una gran importancia como centro distribuidor de bienes entre las regiones áridas y las urbanizadas, sumado al hecho de que se documenta una arquitectura similar (casas aradienses) tanto en Arad como en los asentamientos del desierto, habilita la posibilidad de pensar en que podrían haber intervenido directamente los pastores del Néguev y del Sinaí en su urbanización. De esta manera, Arad sería un caso atípico de urbanismo, en donde la influencia de los pastores móviles habría sido fundamental para su fundación.

Asimismo, podemos comparar la propuesta de Finkelstein (1990) con las ideas formuladas para explicar los asentamientos circulares, o en forma de anillo, que se han desarrollado en el tercer milenio a.C. en Siria, denominados Kranzhügel. Según algunos autores, estos sitios tendrían esta forma debido a que en sus orígenes funcionaban como un centro de almacenaje de bienes en silos, eran viviendas estacionales, o bien pudieron haber sido utilizado con fines políticos, religiosos o de protección (Lyonnet, 2009: 180). Algunas estructuras domésticas de Kranzhügel como las halladas en Mari fueron comparados con las casas aradianas, ya que presentan una arquitectura similar y también se ha sugerido un origen pastoril estacional (Lyonnet, 2009: 184).

De todas maneras. el posible origen pastoril de Arad no ha sido tenido muy en cuenta en las investigaciones, como ya hemos visto. Además, esta postura fue criticada por Rosen (2017: 203), quien la considera una exageración por los contrastes económicos, sociales, arquitectónicos y de la cultura material que existían entre Arad y los pastores. De todas maneras, más allá del origen de Arad, es claro que ésta poseía estrechos vínculos con las poblaciones pastoriles del Néguev y del Sinaí, lo que se observa en los bienes que circularon entre ambas regiones.

\section{Influencia de la urbanización en la periferia y de la periferia en la urbanización}

Como se pudo apreciar en los casos analizados, podría suponerse que las poblaciones pastoriles de las regiones áridas tuvieron algún rol en el proceso de urbanización levantino. Por un lado, se observa la influencia de algunas urbes en la arquitectura de ciertos sitios pastoriles, y la ocupación de asentamientos al mismo tiempo que el desarrollo urbano. Por otro lado, los testimonios de intercambios y subsistencia permiten inferir interacciones que se establecieron entre pastores móviles y los asentamientos urbanos, al mismo tiempo que el incremento de la circulación de bienes, contemporáneo a la urbanización, podría dar cuenta del rol que los pastores tuvieron para el desarrollo de este proceso.

Algunos asentamientos fueron ocupados casi al mismo tiempo que se desarrollaron los primeros asentamientos urbanos (BA IB-II), mientras que otros ya se encontraban habitados, lo que podría indicar la preexistencia de rutas de intercambios, previas al surgimiento de mercados urbanos. En el sur del Sinaí y en el Néguev central se evidencia la influencia de las sociedades urbanas, ya que 
se observa que algunos sitios son ocupados a partir del BA II (contemporáneo al desarrollo de Arad) y que poseen una cultura material muy similar a la de otros sitios del norte del Néguev, especialmente de Arad.

Estos asentamientos temporarios fueron ocupados al mismo tiempo que, por un lado, acontece el repliegue de la influencia egipcia en el sur del Levante, y por el otro, se autonomizan los centros urbanos levantinos, su población comienza a concentrarse en sitios de mayor altura y se amurallan sus asentamientos -BA II- (cf. Campagno, 2010). A partir de la disminución de asentamientos, procesos de ruralización y la crisis de la primera urbanización en el sur del Levante (BA III-IV), con la desaparición de los centros urbanos también desaparecen algunos sitios atribuidos a poblaciones pastoriles, asociadas a ellos en la región -por ej., Camel Site- (cf. Milevski, 2010; Rosen, 2017). Esto podría indicarnos que sitios como los que abordamos tuvieron una función relacionada con los intercambios con estos centros, y que, al caer la demanda de los mercados urbanos, los pastores reorientaron sus actividades económicas.

Se evidencian estructuras defensivas a partir del BA Ib-II, momento en el cual Egipto se repliega de la región, pero también se extienden las actividades de grupos pastoriles: incremento demográfico, productivo y de asentamientos estacionales. Si a esto le sumamos la existencia de abundantes puntas de flechas, cuando ya se practican actividades pastoriles intensivas y se producen intercambios de productos agrícolas con los centros urbanos sedentarios, podríamos pensar que las actividades de caza no han sido su única función. De esta manera, nos preguntamos si existe la posibilidad de que la mayor cantidad de puntas de flechas se deba a conflictos en la región. Quizás, pudo haber existido un incremento de violencia interpersonal por la intromisión, y posterior repliegue, de Egipto en el Levante, al mismo tiempo que se concentraron las poblaciones sedentarias en los centros urbanos ubicados en regiones altas y defensivas.

En el estado actual del conocimiento es difícil demostrar arqueológicamente la dinámica de estos hipotéticos conflictos. Por este motivo, proponemos pensar que pudo existir cierta conflictividad, en la que los grupos pastoriles de las regiones áridas intermedias entre Egipto y el sur del Levante pudieron tener participación. Tanto su modo de vida móvil, como la posesión de armas, ${ }^{15}$ pudo convertirse en un factor militar importante en la resolución de estos conflictos.

15 Dichas armas podrían haber estado compuestas por arcos y flechas, tal como se observa en el registro arqueológico de abundantes puntas de flechas lunares y transversales que se encuentran en estas regiones (Rosen, 2017: 100). Por otra parte, existen representaciones iconográficas posteriores que muestran a grupos caravaneros asiáticos que arriban a Egipto con su familia, bienes para el intercambio y diferentes armas (arcos y lanzas), como se puede observar en la tumba del nomarca Knumhotep II en Beni Hassan (Reino Medio). Más allá de las distancias temporales con nuestro período estudiado, podemos pensar que, para circular las tierras del Sinaí entre el Levante y Egipto, habrían sido necesarios en cualquier período no sólo una buena logística, para aprovisionarse de alimentos necesarios para atravesar el desierto, sino también armas para defenderse de posibles emboscadas. 
Asimismo, ante la expansión de dinámicas estatales egipcias en la región, podríamos pensar que estos grupos pastoriles hayan ejercido resistencia. Las poblaciones timnitas pudieron haber sido en algunos momentos colaboradores (como trasportadores, extractores de metales, mercenarios, etc.), pero también pudieron oponerse a los intentos de imposición de la autoridad estatal, reaccionando violentamente e incluso influenciando en el posterior repliegue egipcio, habiendo migrado a otras regiones o asentándose. En el caso del sur levantino, en un primer momento, centros como Arad establecieron puestos de avanzada en estas regiones periféricas, donde podrían haber encontrado colaboración de los pastores, como en los sitios mencionados. Sin embargo, con el proceso de concentración y amurallamiento, podríamos pensar que la búsqueda de protección pudo deberse a la influencia de una periferia que se estaba volviendo hostil contra su expansión. ${ }^{16} \mathrm{O}$ si no también, estas poblaciones podrían haberse establecido en Arad y haberse urbanizado como consecuencia de un proceso de adaptación y aprovechamiento de las áreas de cultivos locales. Asimismo, podrían haber establecido una "puerta" de entrada al desierto para las redes de intercambio, las que conectaron el resto de las urbes levantinas con las fuentes de materias primas.

\section{Consideraciones finales}

A partir del análisis desarrollado, proponemos algunas consideraciones respecto a la temática abordada:

En primer lugar, creemos que las poblaciones timnitas participaron activamente en las redes de intercambio y de producción de materias primas. $\mathrm{Su}$ modo de vida basado en el pastoralismo móvil, posibilitó la instalación de redes de intercambios con centros urbanos. Teniendo en cuenta que el proceso de urbanización ${ }^{17}$ para llevarse a cabo requirió de condiciones tales como: alimentos para el abastecimiento de su producción; bienes de prestigio para ser acumulados por una élite; materias primas para la producción artesanal por especialistas a tiempo completo; $y$, si consideramos que "la urbanización del BA tiene que ver con un proceso dialéctico de aumento de la circulación de bienes" (Milevski, 2010: 175), podemos pensar que el rol de estos pastores

16 Para ilustrar estos escenarios, en donde la expansión del Estado encuentra resistencia, podemos mencionar la siguiente idea de Deleuze y Guattari: "cuando un Estado no logra estriar su espacio interior o contiguo, los flujos que lo atraviesan adquieren necesariamente el aspecto de una máquina de guerra dirigida contra él, desplegada en un espacio liso hostil o rebelde (incluso si otros Estados pueden introducir en él sus estrías)" (Deleuze y Guattari, 2002: 390). En este sentido, podríamos pensar la movilidad de estos pastores como un elemento de resistencia frente al desarrollo de lógicas de interacción desiguales que generen escenarios de subordinación política, ya que económicamente se puede observar el establecimiento de relaciones centro-periféricas entre Egipto-Levante y urbes levantinas-hinterland rural y desierto.

17 Con las condiciones necesarias nos referimos a algunos de los indicadores propuestos por Gordon Childe (1950) para entender este fenómeno. 
móviles, encargados de los intercambios, fue fundamental para el surgimiento del urbanismo en el sur del Levante.

En segundo lugar, consideramos que el proceso de urbanización modificó los patrones de asentamiento y la producción de estas sociedades pastoriles. Sin embargo, es necesario tener en cuenta que éste no fue un proceso unidireccional. Por el contrario, creemos que los flamantes centros urbanos fueron influenciados por las actividades de los grupos pastoriles. Por un lado, proporcionaron redes de intercambio para la circulación de bienes, y por el otro, su presencia, que podría volverse hostil, pudo haber sido un factor más en las motivaciones que llevaron a que los centros urbanos levantinos desarrollaran estructuras defensivas.

Finalmente, si bien creemos que es necesario continuar indagando sobre este tópico, consideramos que el proceso de urbanización fue multidireccional. Es decir, las rutas de intercambio y el acceso a materias primas exóticas fue necesario para el desarrollo urbano, al mismo tiempo que su demanda modificó los patrones de producción y movilidad pastoril. De esta manera, el proceso de urbanización consistió en un fenómeno derivado del incremento de las interacciones, con consecuencias paralelas, entre sociedades pastoriles y las sociedades urbanas. Asimismo, si bien no existen evidencias suficientes como para afirmar que Arad fue un asentamiento urbano de origen pastoril, sería interesante indagar en las condiciones de su surgimiento comparativamente, con casos en donde se ha establecido un origen pastoril en la urbanización de ciertos sitios (por ej., Siria). Esto implicaría repensar no sólo el rol de los pastores en los procesos de urbanización de la antigüedad, sino también el origen del urbanismo en el Levante como fenómeno exclusivamente sedentario.

\section{Agradecimientos}

Este artículo está basado en una monografía elaborada en el marco del seminario de grado brindado en 2018 por el Dr. Pablo Jaruf (UBA/CONICET), junto a la Lic. M. Belén Daizo (UBA/UNIPE), denominado "La revolución urbana en Siria-Palestina: de las aldeas a las primeras ciudades (ca. 6500-2000 a.C.)". La investigación pudo ser realizada en parte gracias a mi participación en el proyecto UBACYT n $20020160100059 B A$, "Formas de conflicto en las sociedades del Mediterráneo antiguo", dirigido por el Dr. Marcelo Campagno (UBA/CONICET). Una versión preliminar fue presentada en las "Primeras Jornadas de Investigación del Instituto de Historia Antigua Oriental”, realizadas en 2019. Agradezco la invitación de los integrantes del FILOCyT 19-075 "Ciclos de urbanización y dinámicas sociopolíticas en Siria-Palestina: de la Edad del Bronce a la Edad del Hierro (3600-700 a.C.)", con quienes discutí algunos de los puntos tratados. A todos ellos extiendo mis agradecimientos. Por último, agradezco al Dr. Pablo Jaruf por sus valiosos comentarios y observaciones. Todos los errores y omisiones son de mi entera responsabilidad. 


\section{Bibliografía}

"Abe, M. (2008). The Development of Urbanism and Pastoral Nomads in the Southern Levant. Chalcolithic and Early Bronze Age Stone Tool Production Industries and Flint Mines in the Jafr Basin, Southern Jordan. PhD Thesis. Liverpool: University of Liverpool.

»Abu-Azizeh, W. (2013). The South-Eastern Jordan's Chalcolithic - Early Bronze Age Pastoral Nomadic Complex: Patterns of Mobility and Interaction, en: Paléorient 39 (1): 149-176.

"Amiran, R., Beit-Arieh, I. y Glass, J. (1973). The Interrelationship Between Arad and Sites in Southern Sinai in the Early Bronze Age II (preliminary report), en: Israel Exploration Journal 23 (4): 193-197.

»Anfinset, N. (2010). Metal, Nomads and Culture Contact. The Middle East and North Africa. Londres-Oakville: Equinox Publishing.

"Arbuckle, B. S. y Hammer, E. L. (2018). The Rise of Pastoralism in the Ancient Near East, en: Journal of Archaeological Research 27 (3): 391-449.

» Bar-Yosef, O., Belfer-Cohen, A., Goren, A., Hershkovitz, I., Ilan, O., Mienis, H. K. y Sass, B. (1986). Nawamis and Habitation Sites near Gebel Gunna, Southern Sinai, en: Israel Exploration Journal 36 (3-4): 121-167.

»Beit-Arieh, I. (2003). Archaeology of Sinai. The Ophir Expedition. Tel Aviv: Tel Aviv University, Yass Publications in Archaeology.

»Campagno, M. (2010). Centros y periferias en las relaciones entre el valle del Nilo y el Levante meridional en torno del Bronce Antiguo (ca. 3700-2700 a.C.), en: Rivista degli Studi Orientali 83: 189-214.

»Childe, V. G. (1950). The Urban Revolution, en: The Town Planning Review 21 (1): 3-17.

»Daizo, M. B. (2019). Circulación de bienes entre Egipto y el Levante meridional en el IV milenio a. C. hallazgos arqueológicos en Tell el-Farkha y Tel Erani, en: Revista del Instituto de Historia Antigua Oriental 20: 23-51.

»Deleuze, G. y Guattari, F. (2002). Mil Mesetas. Capitalismo y Esquizofrenia. Valencia: Pre-Texto.

» de Miroschedji, P. (1971). L'Époque pré-Urbaine en Palestine, en: Cahiers de la Revue Biblique 48 (191): 235-36.

» de Miroschedji, P. (1986). Ceramiques et mouvements de populations: le cas de la Palestine au Ille millenaire, en: Barrelet, M. T. y Gardin, J. C. (eds.), A propos des interprétations archéologiques de la poterie. París: Editions recherche sur les civilisations (ERC), 10-46.

» de Miroschedji, P. (2009). Rise and Collapse in the Southern Levant in the Early Bronze Age, en: Cardarelli, A., Cazzella, A., Frangipane, M. y Peroni, R. (eds.), Le ragioni del cambiamento / Reasons for Change, Atti del Convegno Internazionale del Dipartimento di Scienze dell'Antichità 15. Roma: Università Sapienza di Roma, 101-129.

»Digard, J. P. (1982). A propósito de los aspectos económicos de la simbiosis nómadas-sedentarios en la antigua Mesopotamia: el punto de vista de un 
antropólogo sobre el Medio Oriente contemporáneo, en: Silva Castillo, J. (ed.), Nómadas y pueblos sedentarios. México D. F.: El Colegio de México, 9-21.

» Finkelstein, I. (1990). Early Arad - Urbanism of the Nomads, en: Zeitschrift des Deutschen Palästina-Vereins 106: 34-50.

» Finkelstein, I. (1995). Living on the Fringe. The Archaeology and History of the Negev, Sinai and Neighbouring Regions in the Bronze and Iron Ages. Sheffield: Sheffield Academic Press.

» Finkelstein, I., Adams, M. J., Dunseth, Z. C. y Shahack-Gross, R. (2018). The Archaeology and History of the Negev and Neighbouring Areas in the Third Millennium BCE: A New Paradigm, en: Tel Aviv 45 (1): 63-88.

»Greenberg, R. (2019). The Archaeology of the Bronze Age Levant. From Urban Origins to the Demise of City-States, 3700-1000 BCE. Cambridge: Cambridge University Press.

» Honeychurch, W. (2015). Inner Asia and the Spatial Politics of Empire: Archaeology, Mobility, and Culture Contact. Nueva York: Springer.

"Honeychurch, W. y Makarewicz, C. A. (2016). The Archaeology of Pastoral Nomadism, en: Annual Review of Anthropology 45 (1): 341-359.

» Horwitz, L. K. y Tchernov, E. (1989). Animal Exploitation in the Early Bronze Age of the Southern Levant: An Overview, en: de Miroschedji, P. (ed.), L'urbanisation de la Palestine à l'âge du Bronze ancien: bilan et perspectives des recherches actuelles (BAR International Series 527). Oxford: Archaeopress, 279-296.

" Jaruf, P. (2019). Del Calcolítico al Bronce Antiguo en Siria-Palestina: revisando el modelo de la segunda urbanización, en: Claroscuro. Revista del Centro de Estudios sobre Diversidad Cultural 18 (2): 1-28.

»Kempinski, A. (1978). The Rise of an Urban Culture: The Urbanization of Palestine in the Early Bronze Age. Jerusalén: Israel Ethnographic Society.

» Kenyon, K. (1960). Archaeology in the Holy Land. Londres: E. Benn.

" Khazanov, A. M. (1984). Nomads and the Outside World. Cambridge: Cambridge University Press.

»Levy, T. E. (1992). Transhumance, Subsistence, and Social Evolution in the Northern Negev Desert, en: Bar-Yosef, O. y Khazanov, A. (eds.), Pastoralism in the Levant. Archaeological Materials in Anthropological Perspectives. Madison: Prehistory Press, 65-83.

" Lyonnet, B. (2009). Who Lived in the Third-Millennium "Round Cities" of Northern Syria?, en: Szuchman, J. (ed.), Nomads, Tribes, and the State in the Ancient Near East. Chicago: The University of Chicago, 179-201.

»Milevski, I. (2010). Centros urbanos y periferias en la Edad del Bronce Antiguo sud-levantina, en: Rivista degli Studi Orientali 83: 163-83.

» Milevski, I. (2016). Intercambios de productos en el Levante meridional durante el Bronce Antiguo. Una perspectiva marxista. Barcelona: Bellaterra.

» Oren, E. D. (1986). Early Bronze Age Settlement in Northern Sinai: A Model for Egypto-Canaanite Interconnections, en: de Miroschedji, P. (ed.), L'urbanisation de la Palestine à l'âge du Bronze ancien: bilan et perspectives des recherches actuelles (BAR International Series 527). Oxford: Archaeopress, 389-407.

"Porter, A. (2012). Mobile Pastoralism and the Formation of Near Eastern Civilizations. Nueva York: Cambridge University Press. 
»Potts, D. T. (2014). Nomadism in Iran: From Antiquity to the Modern Era. Oxford: Oxford University Press.

»Rosen, S. A. (2003). Early Multi-Resource Nomadism: Excavations at the Camel Site in the Central Negev, en: Antiquity 77: 749-760.

"Rosen, S. A. (2017). Revolutions in the Desert. The Rise of Mobile Pastoralism in the Southern Levant. Nueva York-Londres: Routledge.

»Rowton, M. B. (1982). Factores económicos y políticos en el nomadismo antiguo, en: Silva Castillo, J. (ed.), Nómadas y pueblos sedentarios. México D. F.: El Colegio de México, 21-34.

"Saidel, B. A. y Haiman, M. (2014). Excavation in the Western Negev Highlands Results of the Negev Emergency Survey 1978-89 (BAR International Series 2684). Oxford: Archaeopress.

"Salzman, P. (2004). Pastoralists: Equality, Hierarchy, and the State. Boulder: Westview.

»Storey, G. R. (2006). Urbanism in the Preindustrial World. Tuscaloosa: The University of Alabama Press.

»Tallet, P. (2010). Le roi Den et les lountiou: les Égyptiens au Sud-Sinaï sous la 1re dynastie, en: Archéo-Nil 20: 97-105.

" van den Brink, E. C. M. y Levy, T. E. (eds.) (2002). Egypt and the Levant. Interrelations from the 4 th through the Early 3 rd Millennium B.C.E. Londres-Nueva York: Leicester University Press.

"Wendrich, W. y Barnard, H. (2008). The Archaeology of Mobility: definitions and research approaches, en: Wendrich, W. y Barnard, H. (eds.), The Archaeology of Mobility. Old World and New World Nomadism. Los Ángeles: Cotsen Institute of Archaeology, University of California, 1-16.

» Yoffee, N. (2005). Myths of the Archaic State: Evolution of the Earliest Cities, States and Civilizations. Cambridge: Cambridge University Press.

»Zeder, M. (1991). Feeding Cities: Specialized Animal Economy in the Ancient Near East. Washington DC: Smithsonian Institution Press. 